\title{
La luz a Antioquia llegó desde Austria y perdura
}

\author{
Francisco Medina-Estrada ${ }^{1}$, Jorge Garcia-Sucerquia ${ }^{2, *}$ \\ ${ }^{1}$ Universidad de Antioquia \\ ${ }^{2}$ Universidad Nacional de Colombia-Sede Medellín - Escuela de Física
}

\begin{abstract}
Resumen
Se presenta en este artículo un recuento resumido desde la perspectiva de los autores de la forma como se inició el estudio de la luz en Antioquia y como se mantiene. Se hace énfasis en las contribuciones a este proceso por parte del Prof. Dr. Peter Barlai, quien llegó desde Austria al principio de los años 70, estableció las bases de los grupos de óptica de las dos Universidades más importantes de la región antioqueña. Se mencionan además, los aportes a la constitución de los demás grupos que estudian la luz en Antioquia por parte de instituciones nacionales e internacionales. Se muestra la conexión existente entre el trabajo actual de los grupos más representativos y los estudios originales en el campo de la luz en la región.
\end{abstract}

Palabras clave: óptica en Antioquia, Peter Barlai.

Light came to Antioquia from Austria and last

\begin{abstract}
In this paper, we present a condensed summary on the onset of the study of light in Antioquia and how it has prevailed until today. We highlight the contributions of Prof. Dr. Peter Barlai since he arrived from Austria in the early seventies, and how he laid the basis for the establishment of the optics groups of the two leading Universities of Antioquia. In addition, we explain the involvement of national and international institutes in the formation of the optics groups of Antioquia, as well as the link between the original onset of the study of light and the current interest of the leading groups.
\end{abstract}

Key words: Optics in Antioquia, Peter Barlai.

\section{Introducción}

La celebración de un evento tan importante como el Año Internacional de la Luz 2015 trajo a nuestro país eminentes personajes de la escena mundial en tema relacionados con la luz, entre los que se encontraban premios Nobel en física, e incentivó a personalidades del más alto nivel gubernamental a interesarse por el que hacer con la luz. El Presidente de la República y la Ministra de Educación, se animaron a mirar hacia los que estudiamos este temática y permitió a una gran cantidad de ciudadanos que leen la hora en su reloj de pulsera, o en su teléfono inteligente, a acercarse a temas tan exóticos como relojes atómicos con exactitud en el rango de las oscilaciones atómicas o el entrelazamiento cuántico entre partículas; a algunos nos permitió distraernos de nuestra agitada vida para atender a tan ilustres visitantes y para preguntar por temas como: ¿de dónde surgió el interés por la luz en nuestro entorno?, quizás con el ánimo de ofrecer un reconocimiento hasta ahora negado a todos aquellos que nos inculcaron el interés por estudiar la luz, entendida ésta como esa oscilación electromagnética que nos maravilla.

Atendiendo a esta inquietud y en el inigualable marco de la celebración del Año Internacional de la Luz 2015, queremos hacer un recuento de cómo llegó la luz a Antioquia para perdurar hasta nuestros días, entendiendo esta frase como la forma en la cual se estableció el estudio de las ondas electromagnéticas.

\section{El origen}

Al ingresar al cuarto de revelado fotográfico del Laboratorio de Óptica de la Universidad de Antioquia, los visitantes preguntan: ¿Quién es él? Se indaga por una copia en tamaño 9"x11" de la fotografía que se muestra abajo. "Es Peter Barlai", es la respuesta del anfitrión.

El departamento de Física de la Universidad en los primeros años de la década de los 70 centraba su labor en ofrecer cursos de servicio para la establecida Facultad de Ingeniería. Se iniciaba de forma incipiente la investigación en dicho departamento con los grupos de investigación en las áreas de Fenomenología de Partículas, Estado Sólido y Biofísica. Con el apoyo de la Organización Internacional para las

\footnotetext{
*Correspondencia:

Jorge Garcia-Sucerquia, jigarcia@unal.edu.co

Recibido: 16 de julio de 2015

Aceptado: 18 de septiembre de 2015
} 


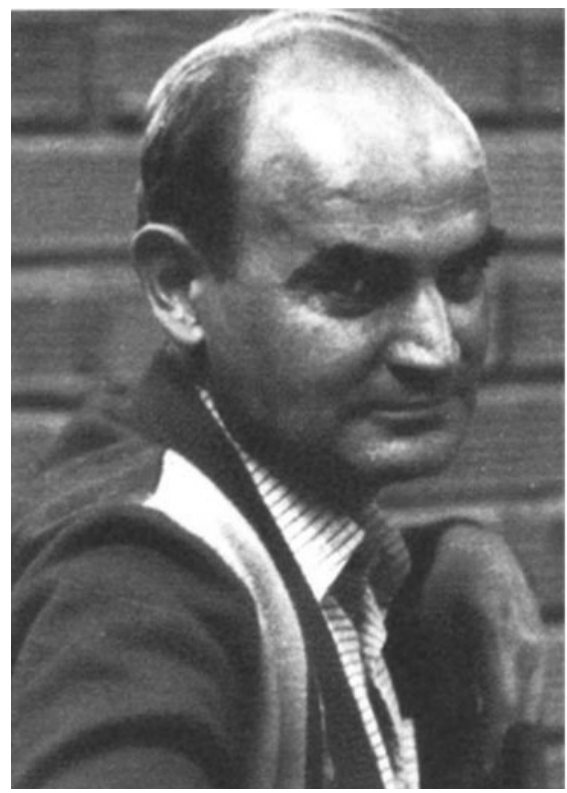

Figura 1. Fotografía del Dr. Peter Barlai, cortesía Grupo de Óptica y Fotónica de la Universidad de Antioquia.

Migraciones, se incorporó a este departamento el Prof. Dr. Peter Barlai (Viena-Austria, agosto 22 de 1939 - AsunciónParaguay enero 20 de 1985), quien dio origen en el año 1972, el grupo de investigación en Óptica con el objetivo de estudiar al grupo de la luz desde diferentes perspectivas.

Bajo la coordinación del Prof. Barlai, los profesores del departamento de Física Lorenzo de la Torre, Francisco Fernando Medina, Orlando Quintero y Fabio Machado, participaron en seminarios en el tema de la luz como por ejemplo holografía (Barlai, 1971a; Barlai, 1971b; Barlai, 1972a), láseres y sus aplicaciones (Barlai, 1971b; Barlai, 1972b), ergodicidad y coherencia (Barlai, 1973a; Barlai, 1971c; Barlai, 1973b); también se contó con la participación de algunos estudiantes de la carrera de Física, los cuales posteriormente se unieron al grupo liderado por el Prof. Barlai. Además de estos seminarios, se iniciaron cursos dirigidos con la participación activa de cada uno de los miembros del grupo y se dio comienzo a la realización de trabajos de grado a nivel de pregrado, formalizando de esta forma la investigación en el grupo de Óptica, principalmente en las temáticas de los seminarios desarrollados. Se trazaron de esta forma las líneas de interés de estudio de la luz en Antioquia que perduran hasta nuestros días.

Entre 1975 y 1977, como coordinador del grupo de Óptica, el Prof. Barlai tuvo una estancia académica en Alemania con el fin de continuar trabajos investigativos en el área de la holografía. Durante este periodo, el Prof. Francisco Fernando Medina asumió la coordinación del grupo, llevando a feliz término trabajos de grado en las áreas de interés del grupo de Óptica.
El Prof. Peter Barlai regresó con nuevas ideas, con lo cual se intensificó el trabajo académico del grupo de Óptica de la Universidad de Antioquia realizando nuevos seminarios, cursos de formación y nuevos proyectos de investigación. Tres años después, se incorporó al grupo el primer doctor nacional en el dominio la luz, el Dr. Eddien Álvarez, quien regresó de Suecia con un doctorado en espectroscopia láser y abrió una nueva línea de trabajo independiente en el campo de la luz en la Universidad.

La consolidación del estudio de la luz en Antioquia se llevó a cabo en la década de los años 80 , con las primeras tesis de Maestría en Física en el área de Óptica. Se registran, por ejemplo, los trabajos de los profesores Francisco F. Medina y Luis Fernando Jaramillo, sobre las funciones de Walsh y la borrosidad de los patrones de Speckles, respectivamente.

De forma paralela, algunos de los egresados de pregrado y maestría en Física con énfasis en Óptica realizaron su formación doctoral en el extranjero. Por ejemplo, los profesores Román Castañeda y Rodrigo Henao, lo hicieron en Alemania y Argentina, respectivamente.

La reincorporación del Prof. Dr. Castañeda a la Universidad Nacional de Colombia Sede Medellín (UN-Med) en 1993 y del Prof. Dr. Henao a la Universidad de Antioquia en 1997, marcó la consolidación formal del estudio de la luz en Antioquia. El Prof. Castañeda formalizó el grupo de Óptica de la UN-Med donde inició de manera decidida el estudio de la coherencia espacial de la luz (Castañeda \& Medina, 1999), la interferometría y los speckles con el apoyo de profesores y estudiantes de dicha Universidad. Por su parte, el Prof. Henao continuo el estudio de los speckles (Henao, et al., 1993; Torroba, Henao, \& Carletti, 1996; Henao, et al., 1997) e inició el estudio del cifrado de información con el uso de la luz (Castañeda, et al., 2001), temas que se añadieron al estudio de la holografía. De forma conjunta estos dos doctores dirigieron al Prof. Francisco Fernando Medina para obtener el primer título de doctor en Física otorgado por la Universidad de Antioquia, que fue precisamente en el marco del estudio de la luz en el campo de la coherencia espacial parcial.

Los doctores en Física Castañeda y Medina, graduados con estudios en temas relacionados con la luz y con relación directa con el Prof. Peter Barlai, lideraron los procesos formativos en el área de Óptica en las dos Universidades más importantes de la región. En la Universidad Pontificia Bolivariana doctores formados en la escuela de la Universidad Industrial de Santander conformaron el grupo de Óptica y Espectroscopia. El grupo de Óptica Aplicada de la Universidad Eafit se conformó bajo el liderazgo del Dr. Luciano Ángel Toro formado en el Centro de Investigaciones Óptica de la Plata, Argentina. A estos grupos se unieron magísteres y doctores que realizaron sus estudios en diferentes instituciones nacionales e internacionales y fortalecieron los grupos de la Universidad Eafit, Universidad 
Pontificia Bolivariana, Instituto Tecnológico Metropolitano (ITM), entre otras. La suma de estos esfuerzos constituyó el factor determinante para consolidar la región antioqueña como líder en el estudio de la luz en nuestro país.

\section{Nuestros formadores}

El interés inicial sembrado por el Prof. Dr. Peter Barlai se fortaleció con los doctores Castañeda y Medina. Formados bajo el amparo del Prof. Barlai, ellos mantuvieron la tradición instaurada por él en el estudio de la coherencia [5-7], los speckles y la holografía (Barlai, 1971a; Barlai, 1971b; Barlai, 1972a). Además, el estudio de la luz en Antioquia ha tenido un importante apoyo por parte de instituciones del ámbito internacional.

Así, a través del Centro Internacional de Física Teórica (ICTP) de la ciudad de Trieste (Italia) se inició una cooperación con la Universidad de Boloña, más exactamente con los profesores Missiroli, Pozzi y Matteucci. Esta cooperación fortaleció de manera decidida las líneas de coherencia y holografía (Matteucci, et al., 1992; Medina, et al., 2004; Garcia-Sucerquia, et al., 2004; Medina \& Pozzi, 1990).

El Centro de Investigaciones Ópticas de La Plata, Argentina (CIOP), en cabeza de los doctores Roberto Torroba, Marcelo Trivi, Héctor Rabal, Lía Zerbino, Enrique Sicre y Mario Garavaglia, marcó el desarrollo de los grupos de investigación en Óptica de las Universidades de Antioquia, Eafit, Politécnico Jaime Isaza Cadavid y Pontificia Bolivariana. Varios de los investigadores de estos grupos recibieron su formación doctoral en dicho centro y continúan manteniendo una estrecha cooperación científica con sus directores de tesis.

En España, la Universidad de Valencia representada por los profesores Pedro Ándres-Bou, Miguel Ándres-Bou, Manuel Martínez-Corral, Genaro Saavedra, la Universidad Jaume I, con los profesores Enrique Tajahuerce y Jesús Lancis y la Universidad de Murcia con el Profesor Pablo Artal, han hecho parte de la consolidación de los grupos de investigación en óptica de la región por medio de la formación de doctores en sus unidades académicas a través de la dirección de doctorandos con co-tutoría así como también de profesores de nuestros grupos de investigación.

Desde Polonia, los profesores Zbigniew Jaroszewicz y Andrzej Kolodziejczyk del instituto de Óptica Aplicada de Varsovia y la Universidad Tecnológica de Varsovia, han dejado un legado importante en el estudio de elementos difractivos diseñados para el mejoramiento del sistema visual humano (Kakarenko, et al., 2015). Así mismo, sus enseñanzas han marcado el desarrollo de grupos de estudio de la luz como el de la Universidad de Antioquia.

Son muchas las instituciones que han contribuido en el proceso de formación de los miembros de los grupos de investigación que estudian la luz en Antioquia. Dalhousie University (Canadá), Pontificia Universidad Católica Do
Rio De Janeiro (Brasil), Danish Institute of Technology (Dinamarca), Florida Atlantic University (Estados Unidos), figuran entre las instituciones que han hecho parte de estos procesos de formación y de alguna forma han contribuido a mantener vivo el interés sembrado por el Prof. Dr. Peter Barlai.

\section{El estudio de la luz se mantiene hasta nuestros días}

El interés por el estudio de la luz, demostrado e inculcado por aquellos que trajeron esta pasión a la región, se ha fortalecido en nuestros días. Grupos consolidados de investigación en las Universidades de Antioquia, Eafit, ITM, Medellín, Nacional de Colombia Sede Medellín, Politécnico Jaime Isaza Cadavid y Pontificia Bolivariana, constituyen el núcleo más sólido para el estudio de la luz en Colombia. La región cuenta en la actualidad con grupos de investigación es este campo con la máxima categoría otorgada por la rectora de la investigación en Colombia Colciencias, trabajando en temas como óptica visual (Kakarenko, et al., 2015), teoría de coherencia (Castañeda, 2014), fotónica y optoelectrónica (Torres, Aristizábal, \& Andrés, 2011; Reyes-Vera, et al., 2012; Reyes-Vera, et al., 2014), vórtices ópticos (Sierra-Sosa, et al., 2013; Angel-Toro, et al., 2013; Angel-Toro, et al., 2012; Londoño, et al., 2015; Rueda, et al., 2013), óptica no-lineal (Serna, et al., 2014), fenómenos ultra-rápidos (Mendoza-Yero, Tajahuerce, et al., 2013; Mendoza-Yero, Calabuig, et al., 2013), microscopía confocal (Garzón, et al., 2008), holografía digital (Hincapie, Herrera-Ramirez, \& Garcia-Sucerquia, 2015; Garcia-Sucerquia, et al., 2005; Velásquez Prieto \& Garcia-Sucerquia, 2006), microscopía holográfica digital (Garcia-Sucerquia, et al., 2006; Sánchez-Ortiga, et al., 2014; Doblas, Sánchez-Ortiga, et al., 2015; Doblas, Hincapie-Zuluaga, et al., 2015), microscopía holográfica digital multiespectral (Garcia-Sucerquia, 2012; MendozaYero, Tajahuerce, et al., 2013), métodos opto-digitales para cifrado de información (Barrera, Vélez, \& Torroba, 2014; Barrera, Mira, \& Torroba, 2013; Barrera, et al., 2012; Barrera \& Torroba, 2009), entre otros.

El sueño de aquellos que iniciaron el estudio de la luz en Antioquia se ha hecho realidad. Varios grupos de la región son referentes internacionales en su dominio de conocimiento. Este liderazgo se ha traducido en el otorgamiento de patentes de invención (Sánchez-Ortiga, et al., 2014; Garcia-Sucerquia, Trujillo, \& Restrepo Agudelo, 2014), producción de los artículos más descargados (Barrera, et al., 2012) y más citados (Check out the Top Cited Articles in Applied Optics, 2013) en revistas de primera línea en el ámbito internacional, realización de conferencias e invitación a participar en los más prestigiosos eventos de la óptica en el mundo, publicación de artículos en las revistas del más alto impacto en la rama de la óptica, el reconocimiento por medio de premios nacionales e internacionales y la formación de estudiantes con proyección nacional e internacional. 
Muchos de los estudiantes formados en nuestras escuelas de estudio de la luz han encontrado acogida en centros de investigación tan prestigiosos como Oxford University, Max Planck Institute o DTU, donde hacen parte de grupos de primera línea mundial. Algunos otros han regresado de sus estudios doctorales así como también estancias posdoctorales en prestigiosos centros del ámbito internacional con el ánimo y la capacidad de establecer programas de investigación de relevancia internacional, demostrando que ese interés por el estudio de la luz que nos llegó a Antioquia desde Austria se ha consolidado y se proyecta como uno de los dominios en los cuales la región se destaca en el contexto latinoamericano, garantizando de esta forma su permanencia a corto y mediano plazo.

\section{Agradecimientos}

Los autores agradecen el apoyo de la Universidad Nacional de Colombia, Proyecto Hermes 19384, el Programa de Internacionalización del Conocimiento y el programa de Jóvenes Investigadores de Colciencias-Universidad Nacional de Colombia código Hermes 28751.

\section{Referencias}

Angel-Toro, L. Sierra-Sosa, D., Tebaldi, M., \& Bolognini, N. (2012). Vortex Metrology Using Fourier Analysis Techniques: Vortex Networks Correlation Fringes. Applied Optics, 51 (30). OSA, 7411-19. doi:10.1364/AO.51.007411.

Angel-Toro, L. Sierra-Sosa, D., Tebaldi, M., \& Bolognini, N. (2013). In-Plane Displacement Measurement in Vortex Metrology by Synthetic Network Correlation Fringes. Journal of the Optical Society of America A, 30 (3). OSA, 462-69. doi:10.1364/JOSAA.30.000462.

Barlai, P. (1971)a). Holographic Reconstruction without Granularity. Naturforsch, 26 1, 1441.

Barlai, P. (1971)b). Information Processing by Holograms. Kybernetik, 2: 78.

Barlai, P. (1971)c). Quantum Theory of Diffraction. Angeu Phys, 31: 82.

Barlai, P. (1972)a). Hologramme Mit Nichtspharischer Reférenzwelle Im Inkoharenten Strahlungsfeld. Z. Naturforsch, 27a: 1777-83.

Barlai, P. (1972)b). Phasenhologramme in Photographischen Emulsionen Mit Hohem Wirkungsgrad Im Blaugriinen Spektralbereich. Z. Naturforsch, 27a: 544.

Barlai, P. (1973)a). Higher-Order Coherence in Optics. Naturforsch, 28a.

Barlai, P. (1973)b). Koharenz Hoherer Ordnung in Der Optik Und Ihre MeBbarkeit. Z. Naturforsch, 28a: 19461950.

Barrera, J., Mira, A., \& Torroba, R. (2013). Optical Encryption and QR Codes: Secure and Noise-Free Information Retrieval. Optics Express, 21 (5). OSA, 5373-78. doi:10. 1364/OE.21.005373.

Barrera, J., Tebaldi, M., Ríos, C., Rueda, E., Bolognini, N., \& Torroba, R. (2012). Experimental Multiplexing of
Encrypted Movies Using a JTC Architecture. Optics Express, 20 (4). OSA, 3388-93. doi:10.1364/OE.20.003388.

Barrera, J., Vélez, A., \& Torroba, R. (2014). Experimental Scrambling and Noise Reduction Applied to the Optical Encryption of QR Codes. Optics Express, 22 (17). OSA, 20268-77. doi:10.1364/OE.22.020268.

Barrera, J., \& Torroba, R. (2009). Efficient Encrypting Procedure Using Amplitude and Phase as Independent Channels to Display Decoy Objects. Applied Optics, 48 (17). OSA, 3120-28. doi:10.1364/AO.48.003120.

Castañeda, R, \& Medina, F. (1999). Moiré Patterns in Spatially Partial Coherent Interference with Non-Regular Gratings. Optik, 110 (3). Elsevier, 123-26.

Castañeda, R. (2014). Electromagnetic Wave Fields in the Microdiffraction Domain. Physical Review A, 89 (1). American Physical Society, 13843. http://link.aps.org/ doi/10.1103/PhysRevA.89.013843.

Castañeda, R, Garcia-Sucerquia, J., Henao, R., \& Trabocchi. O. (2001). Information Encryption through Dyadic Permutations. Optics and Lasers in Engineering, 36 (6): 537-44. doi:10.1016/S0143-8166(01)00079-3.

Check out the Top Cited Articles in Applied Optics. (2013). Optical Society of America.http://www.medellin.unal.edu. co/boletines/images/Top_Cited_Articles_from_Applied_ Optics_2014.pdf.

Doblas, A., Hincapie-Zuluaga, D., Saavedra, G., MartínezCorral, M., \& Garcia-Sucerquia. J. (2015). Physical Compensation of Phase Curvature in Digital Holographic Microscopy by Use of Programmable Liquid Lens. Applied Optics, 54 (16). OSA, 5229-33. doi:10.1364/ AO.54.005229.

Doblas, A., Sánchez-Ortiga, E., Martínez-Corral, M., \& Garcia-Sucerquia, J. (2015). Study of Spatial Lateral Resolution in off-Axis Digital Holographic Microscopy. Optics Communications, 352 (October), 63-69. doi:10. 1016/j.optcom.2015.04.066.

Garcia-Sucerquia, J., Trujillo, C., \& Restrepo, J. (2014). Microscopio Holográfico Digital Sin Lentes (MHDSL) Y Método Para Visualizar Muestras. Colombia: SIC (Colombia).

Garcia-Sucerquia, J. (2012). Color Lensless Digital Holographic Microscopy with Micrometer Resolution. Optics Letters, 37 (10), 1724-26. doi:10.1364/OL.37.001724.

Garcia-Sucerquia, J, Medina, F., \& Matteucci, G. (2004). Optical Tubular Structures Produced by Diffraction of Circular Apertures. Optics and Lasers in Engineering, 42 (1): 61-70. doi:10.1016/S0143-8166(03)00077-0.

Garcia-Sucerquia, J., Herrera-Ramírez, J., \& Velásquez, D. (2005). Reduction of Speckle Noise in Digital Holography by Using Digital Image Processing. Optik - International Journal for Light and Electron Optics, 116 (1): 44-48. doi:10.1016/j.ijleo.2004.12.004.

Garcia-Sucerquia, J., Xu, W., Jericho, S., Klages, P., Jericho, M., \& Kreuzer, H. (2006). Digital in-Line Holographic Microscopy. Appl. Opt., 45 (5). OSA, 836-50. 
Garzón, J., Gharbi, T., \& Meneses, J. (2008). Real Time Determination of the Optical Thickness and Topography of Tissues by Chromatic Confocal Microscopy. Journal of Optics A: Pure and Applied Optics, 10 (10): 104028. http:// stacks.iop.org/1464-4258/10/i=10/a=104028.

Henao, R., Medina, F., Rabal, H., \& Trivi, M. (1993). ThreeDimensional Speckle Measurements with a Diffraction Grating. Applied Optics, 32 (5). OSA, 726-29. doi:10.1364/ AO.32.000726.

Henao, R, Rabal, H., Tagliaferri, A., \& Torroba, R. (1997). Determination of the Zero-Order Fringe Position in Digital Speckle Pattern Interferometry. Applied Optics, 36 (10). OSA, 2066-69. doi:10.1364/AO.36.002066.

Hincapie, D., Herrera-Ramirez J., \& Garcia-Sucerquia, J. (2015). Single-Shot Speckle Reduction in Numerical Reconstruction of Digitally Recorded Holograms. Optics Letters, 40 (8). OSA, 1623-26. doi:10.1364/OL.40.001623.

Kakarenko, K., Ducin, I., Grabowiecki,K., Jaroszewicz, Z., Kolodziejczyk, A., Mira-Agudelo, A., Krzysztof Petelczyc, Składowska, A., \& Sypek, M. (2015). Assessment of Imaging with Extended Depth-of-Field by Means of the Light Sword Lens in Terms of Visual Acuity Scale. Biomedical Optics Express, 6 (5). OSA, 1738-48. doi:10.1364/BOE.6.001738.

Londoño, N, Rueda,E., Gómez, J., \& Lencina, A. (2015). Generation of Optical Vortices by Using Binary Vortex Producing Lenses. Applied Optics, 54 (4). OSA, 796-801. doi:10.1364/AO.54.000796.

Matteucci, G., Medina, F. \& Pozzi, G. (1992). Electron-Optical Analysis of the Electrostatic Aharonov-Bohm Effect. Ultramicroscopy, 41 (4): 255-68. doi:10.1016/0304-3991(92) 90205-X.

Medina, F., \& Pozzi, G. (1990). Spatial Coherence of Anisotropic and Astigmatic Sources in Interference Electron Microscopy and Holography. Journal of the Optical Society of America A, 7 (6). OSA, 1027-33. doi:10.1364/JOSAA.7.001027.

Medina, F., Garcia-Sucerquia, J., Castañeda, R., \& Matteucci, G. (2004). Angular Criterion to Distinguish between Fraunhofer and Fresnel Diffraction. Optik - International Journal for Light and Electron Optics, 115 (11-12): 547-52. doi:10.1078/0030-4026-00547.

Mendoza-Yero, O., Calabuig, A., Tajahuerce, E., Lancis, J., Andrés, P., \& Garcia-Sucerquia, J. (2013). Femtosecond Digital Lensless Holographic Microscopy to Image Biological Samples. Optics Letters, 38 (17). OSA, 3205-7.

Mendoza-Yero, O., Tajahuerce, E., Lancis, J., \& GarciaSucerquia, J. (2013). Diffractive Digital Lensless Holographic Microscopy with Fine Spectral Tuning. Optics Letters, 38 (12): 2107-9.
Reyes-Vera, E, Torres, P., Chesini, G., \& Cordeiro, C. (2012). Temperature Sensitivity of Photonic Crystal Fiber with Integrated Electrodes. In Press Opt Express,.

Reyes-Vera, E., Gómez-Cardona, N., Chesini, G, Cordeiro, C., \& Torres, P. (2014). Temperature Sensibility of the Birefringence Properties in Side-Hole Photonic Crystal Fiber Filled with Indium. Applied Physics Letters, 105 (20): -. doi:http://dx.doi.org/10.1063/1.4902157.

Rueda, E, Muñetón, D., Gómez, J., \& Lencina, A. (2013). High-Quality Optical Vortex-Beam Generation by Using a Multilevel Vortex-Producing Lens. Optics Letters, 38 (19). OSA, 3941-44. doi:10.1364/OL.38.003941.

Sánchez-Ortiga, E., Doblas, A., Saavedra, G., MartínezCorral, M, \& Garcia-Sucerquia, J. (2014). Microscopio, Método Y Programa de Ordenador Para La Obtención de Imágenes Cuantitativas de Fase Por Medio de Microscopía Holográfica Digital, Y Kit Para Adaptar Un Microscopio Óptico. España: Oficna Española de Patentes.

Sánchez-Ortiga, E., Doblas, A., Saavedra, G., Martínez-Corral, M. \& Garcia-Sucerquia, J. (2014). Off-Axis Digital Holographic Microscopy: Practical Design Parameters for Operating at Diffraction Limit. Applied Optics, 53 (10). OSA, 2058-66. doi:10.1364/ao.53.002058.

Serna, J., Hamad, A., Garcia, H., \& Rueda, E. (2014). Measurement of Nonlinear Optical Absorption and NonLinear Optical Refraction in CdS and ZnSe Using an Electrically Focus-Tunable Lens. In 12th International Conference on Fiber Optics and Photonics, T2C.2. OSA Technical Digest (online). Kharagpur : Optical Society of America. doi:10.1364/PHOTONICS.2014.T2C.2.

Sierra-Sosa, D., Angel-Toro, L., Bolognini, N., \& Tebaldi, M. (2013). Novel Vortex-Transform for High Frequency Modulated Patterns. Optics Express, 21 (20). OSA, 2370611. doi:10.1364/OE.21.023706.

Torres, P., Aristizábal, V., \& Andrés, M. (2011). Modeling of Photonic Crystal Fibers from the Scalar Wave Equation with a Purely Transverse Linearly Polarized Vector Potential. J. Opt. Soc. Am. B, 28 (4). OSA, 787-91. http://josab.osa.org/ abstract.cfm?URI=josab-28-4-787.

Torroba, R., Henao, R., \& Carletti, C. (1996). Digital Polarization-Encoding Technique for Optical Logic Operations. Optics Letters, 21 (23). OSA, 1918-20. doi:10.1364/OL.21.001918.

Velásquez, D., \& Garcia-Sucerquia, J. (2006). ThreeDimensional Surface Contouring of Macroscopic Objects by Means of Phase-Difference Images. Applied Optics, 45 (25): 6381-87. 